\title{
Editorial
}

\section{Concrete Durability and Steel Corrosion}

\author{
Seung-Jun Kwon, ${ }^{1}$ Han-Seung Lee, ${ }^{2}$ Behrouz Shafei, ${ }^{3}$ Yuyin Wang, ${ }^{4}$ and Velu Saraswathy \\ ${ }^{1}$ Hannam University, Daejeon, Republic of Korea \\ ${ }^{2}$ Hanyang University, ERICA Campus, Ansan, Republic of Korea \\ ${ }^{3}$ Iowa State University, Ames, IA, USA \\ ${ }^{4}$ Harbin Institute of Technology, Harbin, China \\ ${ }^{5}$ Central Electrochemical Research Institute, Tamil Nadu, India \\ Correspondence should be addressed to Seung-Jun Kwon; jjuni98@hannam.ac.kr
}

Received 10 October 2016; Accepted 11 October 2016

Copyright (C) 2016 Seung-Jun Kwon et al. This is an open access article distributed under the Creative Commons Attribution License, which permits unrestricted use, distribution, and reproduction in any medium, provided the original work is properly cited.

Durability problem which can seriously affect safety of concrete structure has been issued and its significance is increasing. In order to understand and report the issue, four guest editors participated in the works for this special issue.

For almost 1 year of devotion, we are pleased to inform the publication of this special issue with valuable 17 papers through strict and fair reviews. This special issue contains numerical modeling on chloride attack and carbonation, profound investigation of test results, identification of deteriorating mechanism, and NDT-based assessment for deterioration detection.

Among the 17 papers, 3 papers are for applications of NDT to durability research utilizing IR thermography ("Experimental Study on Detection of Deterioration in Concrete Using Infrared Thermography Technique"), electromagnetic properties ("Effect of Chlorides on Conductivity and Dielectric Constant in Hardened Cement Mortar: NDT for Durability Evaluation"), and impact echo method ("Evaluation of Subsurface Damage in Concrete Deck Joints Using Impact Echo Method"). These clearly show that evaluation results of NDT can quantify deterioration and also can be effective for evaluation or prediction of durability performance.

Five papers deal with evaluation of durability performance regarding chloride attack and carbonation. In the works, valuable researches are performed on quantitative corrosion resistance in concrete with steel fibers ("Durability of Steel Fibres Reinforcement Concrete Beams in Chloride
Environment Combined with Inhibitor"), investigation of influencing parameters to carbonation ("Estimation of Concrete Carbonation Depth Considering Multiple Influencing Factors on the Deterioration of Durability for Reinforced Concrete Structures"), structural performance in corroded steel plate ("Experimental Research on Hysteretic Characteristics of Steel Plates Artificially Corroded by Neutral Salt Spray"), quantification of test method for combined deteriorations ("An Accelerated Test Method of Simultaneous Carbonation and Chloride Ion Ingress: Durability of Silica Fume Concrete in Severe Environments"), and chloride behavior evaluation in concrete with various supplementary materials ("Diffusion Decay Coefficient for Chloride Ions of Concrete Containing Mineral Admixtures”).

Researches on numerical modeling always provide a chance to overcome many difficulties in experiments. Five papers are published for corrosion propagation in file foundation and bridge pier ("Mathematical Model of Homogeneous Corrosion of Steel Pipe Pile Foundation for Offshore Wind Turbines and Corrosive Action" and "Computational Modelling Strategies for Nonlinear Response Prediction of Corroded Circular RC Bridge Piers"), modeling on hardening process and strength evaluation in concrete with supplementary materials ("A New Microstructure Development Model for the Evaluation of Concrete Setting Time" and "A Microstructure Based Strength Model for Slag Blended Concrete with Various Curing Temperatures"), and crack 
effect on chloride behavior in concrete as porous media ("Simulation of Chloride Diffusion in Cracked Concrete with Different Crack Patterns").

Two papers dealing with field investigation results report phenomena of corrosion progress and chloride penetration in a bridge structure ("Diagnostics of Corrosion on a Real Bridge Structure") and tunnel concrete ("Chloride Transport in Undersea Concrete Tunnel"), respectively. The results from field or long exposure conditions give us not only information on how much deteriorated a structure has been so far but also on how much improved or worsened a structure will be from now.

Another 2 papers are for structural performance in retrofitted concrete member ("Calculation of Axial Compression Capacity for Square Columns Strengthened with HPFL and BSP") and fracture performance ("Research on the Fracture Properties and Modification Mechanism of Polyester Fiber and SBR Latex Modified Cement Concrete") in LMC (Latex Modified Concrete) which are enhanced by polymer. The works are not for deterioration evaluation but contain valuable results for readers on structural performance in concrete with repaired and rehabilitated conditions.

Through the papers of special issues, we hope the researches on concrete durability are more activated and contribute to academic and industrial fields.

\title{
Acknowledgments
}

The lead guest editor appreciates the guest editors and the reviewers who make a great effort from selection of topic to reviewing process in the schedule.

\author{
Seung-Jun Kwon \\ Han-Seung Lee \\ Behrouz Shafei \\ Yuyin Wang \\ Velu Saraswathy
}



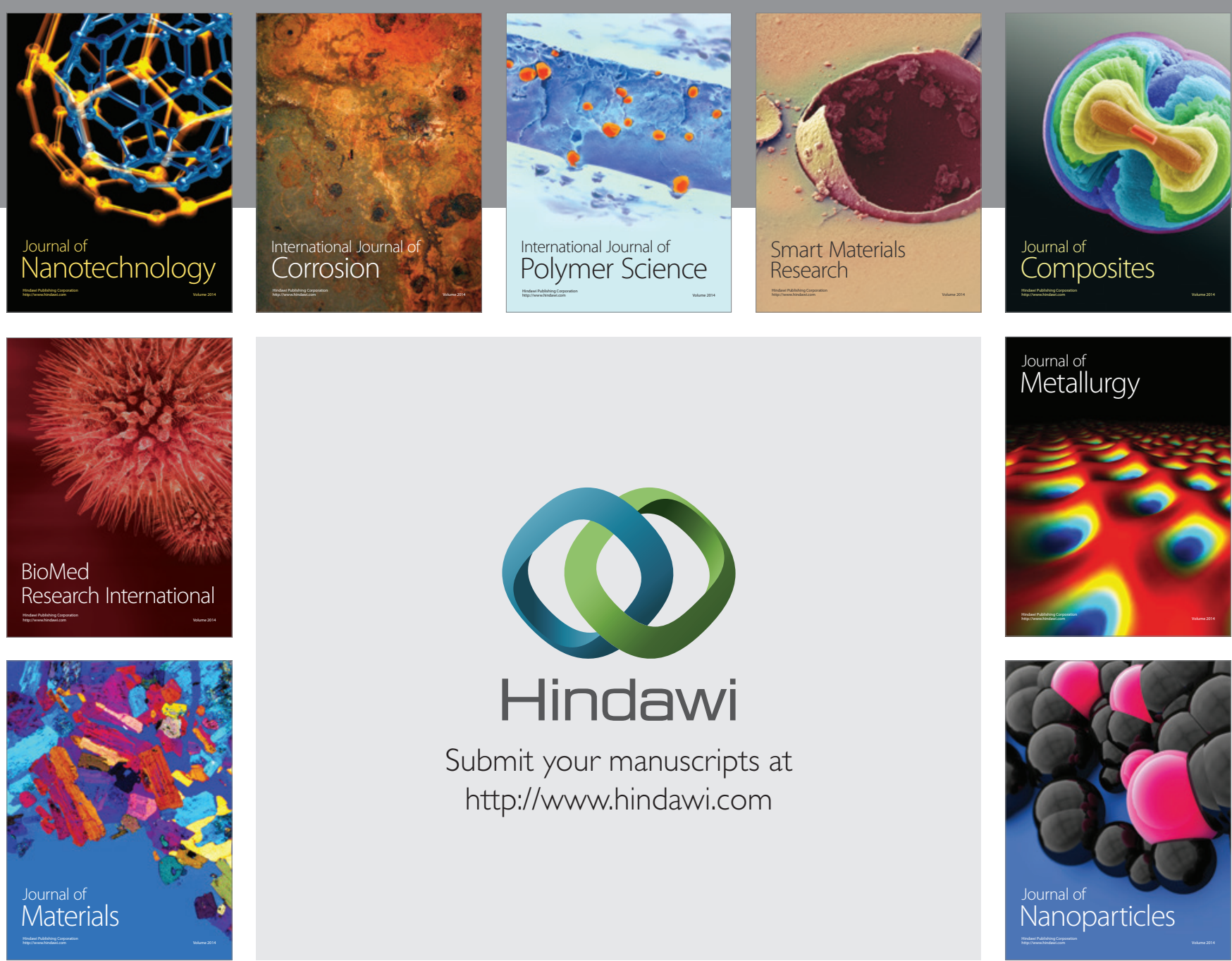

\section{Hindawi}

Submit your manuscripts at

http://www.hindawi.com

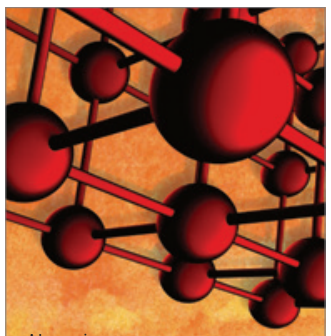

Materials Science and Engineering
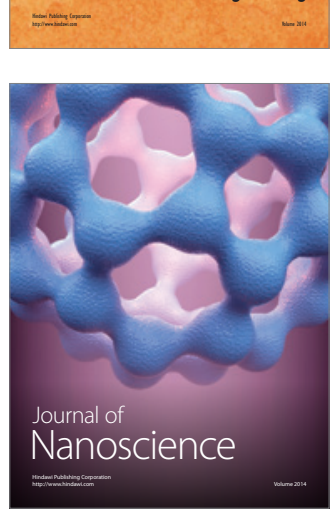
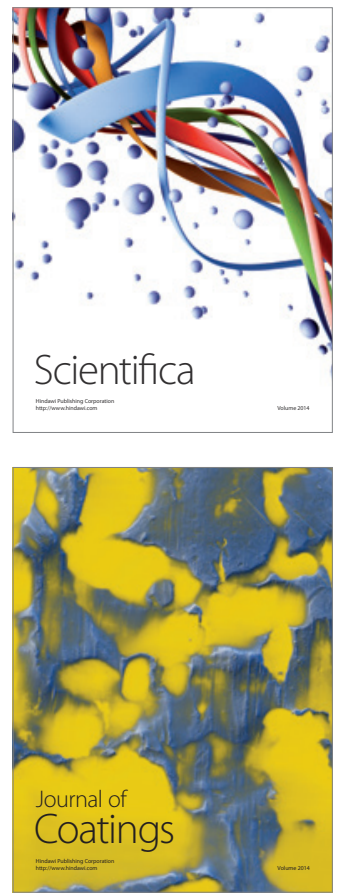
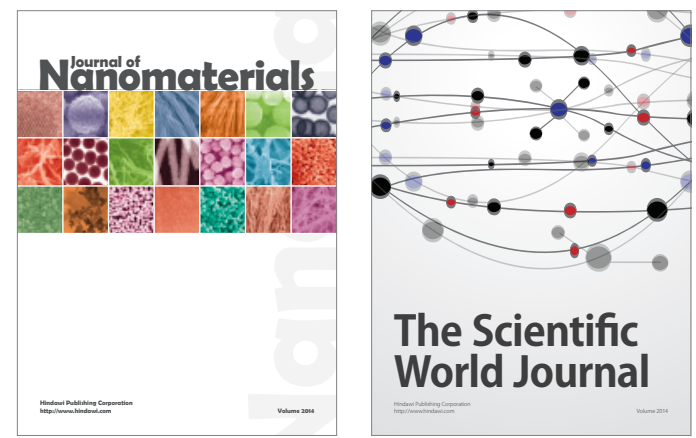

The Scientific World Journal
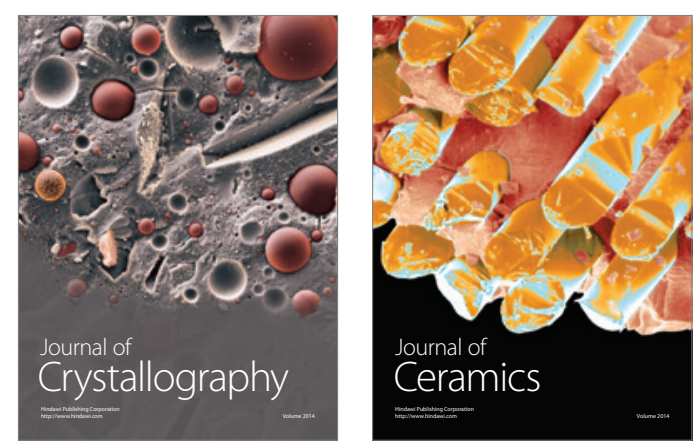
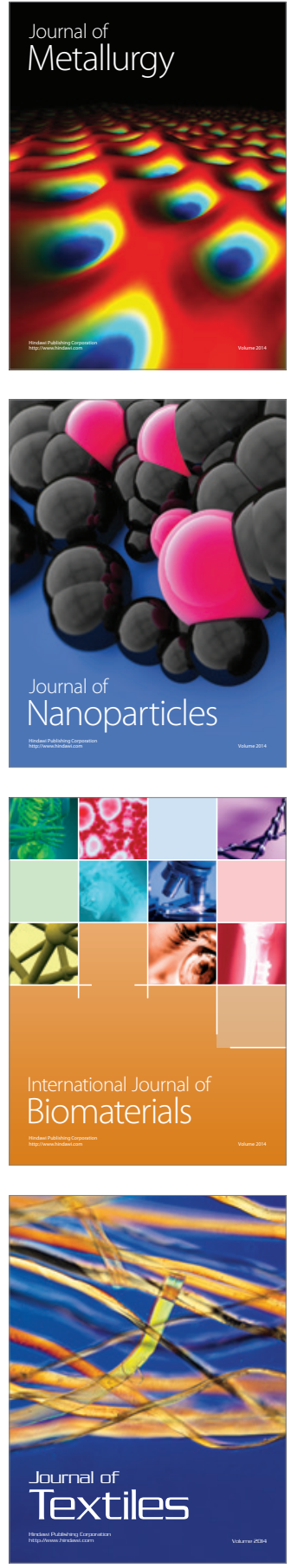